Please cite this paper as follows:

Han-Chin Wu, Hong-Ki Hong, and Ya-Po Shiao, Anisotropic Plasticity with Application to Sheet Metals, International Journal of Mechanical Sciences, Vol.41, pp.703-724, 1999. 


\title{
Anisotropic plasticity with application to sheet metals
}

\author{
Han-Chin Wu ${ }^{\mathrm{a}}$, Hong-Ki Hong ${ }^{\mathrm{b}}$, Ya-Po Shiao ${ }^{\mathrm{b}}$ \\ ${ }^{a}$ Department of Civil and Environmental Engineering, The University of lowa, Iowa City, IA 52242, U.S.A. \\ 'Department of Civil Engineering, National Taiwan University, Taipei, Taiwan, ROC
}

Received 2 December 1996; and in revised form 9 February 1998

\begin{abstract}
Hill's 1948 anisotropic theory of plasticity is extended to include the concept of isotropic-kinematic hardening. The "anomalous" effect can be accounted for by kinematic hardening. It is shown that the quadratic yield function can be used for sheet metals irrespective of its plastic strain ratio $R$. It is further shown that effects of thickness reduction due to further rolling may be accounted for by kinematic hardening. (C) 1999 Elsevier Science Ltd. All rights reserved.
\end{abstract}

\section{Notation}

$x, y, z \quad$ rolling, transverse, normal directions

$x^{\prime} \quad$ axial direction of a tension specimen

$y^{\prime} \quad$ transverse direction of a tension specimen

$\phi \quad$ yield function

$a, b, m \quad$ coefficients of Hill's 1990 yield function

$p, q \quad$ coefficients of Hill's 1993 yield function

$F, G, H, N \quad$ coefficients of Hill's 1948 yield function

$\sigma_{x}, \sigma_{y}, \sigma_{x y} \quad$ stress components

$\sigma_{1}, \sigma_{2}, \sigma_{3} \quad$ principal stresses

$\bar{\sigma} \quad$ effective stress

$\sigma_{x}^{\mathrm{Y}}, \sigma_{y}^{\mathrm{Y}}, \sigma_{x y}^{\mathrm{Y}} \quad$ yields stresses

$\sigma_{u} \quad$ tensile yield stress

$\sigma_{b}$ or $\sigma_{\mathrm{B}}$ or $\sigma_{\mathrm{B}}^{\mathrm{Y}}$ equibiaxial yield stress

$\tau, \sigma_{x y}^{\mathrm{Y}} \quad$ yield stress under pure shear parallel to the orthotropic axes

$S, \sigma_{x} \quad$ tensile stress and tensile yield stress in tension specimen

$R, R_{x} \quad$ plastic strain ratio

$\alpha \quad$ angle of the tension specimen measured from the $x$-axis

$R_{0}, R_{45}, R_{90} \quad$ plastic strain ratios when $\alpha=0^{\circ}, 45^{\circ}, 90^{\circ}$

$\mathrm{d} \varepsilon_{x}^{\mathrm{p}}, \mathrm{d} \varepsilon_{y}^{\mathrm{p}}, \mathrm{d} \varepsilon_{x y}^{\mathrm{p}} \quad$ plastic strain increments

$f$

isotropic hardening function

0020-7403/99/S - see front matter (C) 1999 Elsevier Science Ltd. All rights reserved.

PII: S $0020-7403(98) 00033-2$ 
$r_{x}, r_{y}, r_{x y}$

$r_{x}^{0}, r_{y}^{0}, r_{x y}^{0}$

$\theta^{0}, r_{\max }^{0}, r_{\min }^{0}$

$\bar{r}_{x}, \bar{r}_{y}, \bar{r}_{x y}$

$\bar{\theta}, \bar{r}_{\max }, \bar{r}_{\min }$ back stress, kinematic hardening

first stage back stress

principal direction and values of first stage back stress

second stage back stress

principal direction and values of second stage back stress

\section{Introduction}

Hill's 1948 quadratic yield criterion [1] for anisotropic sheet metal is simple and is widely used in application. It is generally satisfactory for predicting the sheet metal behavior for $R \geqslant 1$, where $R$ is the plastic strain ratio of the transverse to the thickness strain and it specifies anisotropy. In the case of $R<1$, "anomalous behavior" has been observed. In this case, the yield stress in the equibiaxial tension test is higher than that for the uniaxial tension, and Hill's 1948 quadratic yield criterion does not predict this behavior. The case of $R<1$ is important, because it includes such important metals as aluminum.

Numerous non-quadratic yield functions have been proposed in the literature to account for the case of $R<1$. They are generally quite complex and difficult to apply and they do not describe the behavior characterized by effectively equal yield points under tension in the rolling and transverse directions, allied to markedly different strain-ratios [2]. It is not desirable from the viewpoint of unified approach that a quadratic yield function works for $R \geqslant 1$ and a non-quadratic yield function works for $R<1$. Furthermore, for the ease of application, it is also not desirable to have yield functions for anisotropic materials drastically different in form (with higher powers) than those for the same materials in the isotropic state. In the isotropic state, most engineering materials have a quadratic initial yield function. A rule of evolution from the quadratic function to the nonquadratic function would have to be furnished so that the theory can account for the case of initially isotropic materials gradually becoming anisotropic due to deformation.

The objective of this research is to establish Hill's 1948 quadratic yield criterion as the one that is applicable to all sheet metals, irrespective of its $R$ ratio. Furthermore, it will be shown that the proposed theory can be used to describe the plastic behavior for cold-rolled metal sheets subjected to various degrees of thickness reduction.

In Section 2, a literature survey is given for the subject. An extension of Hill's 1948 anisotropic theory of plasticity is given in Section 3. In Section 4, the equations for anisotropic sheet with thickness reduction by further rolling are derived. Finally, three cases of applications are described in Section 5. These three cases include all values of $R$, greater than, equal to, or less than one.

\section{State of knowledge in the field}

\subsection{The yield function}

In the case of rolled sheet metal, when principal axes of anisotropy are the axes of reference, the yield function $\phi$ proposed by Hill [1] has been widely used. The yield function $\phi$ is

$$
2 \phi=(G+H) \sigma_{x}^{2}-2 H \sigma_{x} \sigma_{y}+(F+H) \sigma_{y}^{2}+2 N \sigma_{x y}^{2}=1,
$$


where $x$ denotes the rolling direction and $y$ the transverse direction; $\left(\sigma_{x}, \sigma_{y}, \sigma_{x y}\right)$ are the in-plane components of Cauchy stress; and the out-of-plane components are considered to be zero. The coefficients $F, G, H$, and $N$ specify the anisotropy of the metal sheet. This quadratic form (and its variations such as the one for planar isotropy) has been repeatedly used in applications. It is generally satisfactory for predicting the sheet metal behavior for $R \geqslant 1$.

In the case of $R<1$, "anomalous behavior" has been observed by Pearce [3] and Woodthorpe and Pearce [4] for commercially pure aluminum sheet. In this case, the yield stress in the equibiaxial tension test is higher than that for the uniaxial tension, and Eq. (1) does not predict this behavior. To accommodate the "anomalous behavior", Hill $[5,6]$ proposed a number of possible generalizations of the quadratic yield function. These yield functions are generally more complex and are non-quadratic with additional anisotropic parameters.

Hill (1979):

$$
\begin{gathered}
F\left|\sigma_{2}-\sigma_{3}\right|^{m}+G\left|\sigma_{3}-\sigma_{1}\right|^{m}+H\left|\sigma_{1}-\sigma_{2}\right|^{m}+L\left|2 \sigma_{1}-\sigma_{2}-\sigma_{3}\right|^{m} \\
+M\left|2 \sigma_{2}-\sigma_{3}-\sigma_{1}\right|^{m}+N\left|2 \sigma_{3}-\sigma_{1}-\sigma_{2}\right|^{m}=1 .
\end{gathered}
$$

Hill (1990):

$$
\begin{aligned}
\mid \sigma_{1}+ & \left.\sigma_{2}\right|^{m}+\left(\sigma_{\mathrm{B}}^{m} / \tau^{m}\right)\left|\sigma_{1}-\sigma_{2}\right|^{m} \\
& +\left|\sigma_{1}^{2}+\sigma_{2}^{2}\right|^{(m / 2)-1}\left\{-2 a\left(\sigma_{1}^{2}-\sigma_{2}^{2}\right)+b\left(\sigma_{1}-\sigma_{2}\right)^{2} \cos 2 \alpha\right\} \cos 2 \alpha=\left(2 \sigma_{\mathrm{B}}\right)^{m},
\end{aligned}
$$

where $F, G, H, L, M, N$ and $m$ (with $m>1$, integer or non-integer) are material coefficients; $\sigma_{1}$, $\sigma_{2}$ and $\sigma_{3}$ are principal stresses and are expressed in the orthotropic symmetry axes $x, y$ and $z ; \sigma_{\mathrm{B}}$ is the equibiaxial tensile yield stress and $\tau$ is the yield stress under pure shear parallel to the orthotropic axes. In addition,

$$
a=(F-G) /(F+G) \text { and } b=(F+G+4 H-2 N) /(F+G) .
$$

Note that, in Eq. (2), the principal loads must coincide with the axes of orthotropy, whereas in Eq. (3) they can have any orientation. Furthermore, Eq. (3) is restricted to plane stress.

A yield function was proposed by Hosford [7] in the form of

$$
F\left|\sigma_{1}-\sigma_{2}\right|^{m}+G\left|\sigma_{2}-\sigma_{3}\right|^{m}+H\left|\sigma_{3}-\sigma_{1}\right|^{m}=1
$$

where $m$ is 6 or 8 according to whether the crystal grains are body-centered or face-centered cubic, and the function does not include the anomaly.

Other non-quadratic forms have been proposed in the literature. Gotoh [8] proposed

$$
\begin{gathered}
\bar{\sigma}^{4}=C_{1} \sigma_{x x}^{4}+C_{2} \sigma_{x x}^{3} \sigma_{y y}+C_{3} \sigma_{x x}^{2} \sigma_{y y}^{2}+C_{4} \sigma_{x x} \sigma_{y y}^{3}+C_{5} \sigma_{y y}^{4} \\
+\sigma_{x y}^{2}\left(C_{6} \sigma_{x x}^{2}+C_{7} \sigma_{x x} \sigma_{y y}+C_{8} \sigma_{y y}^{2}\right)+C_{9} \sigma_{x y}^{4}
\end{gathered}
$$

where $\bar{\sigma}$ is the effective stress; $C_{\mathrm{i}}$ are constants. Barlat and Lian [9] used the following criterion:

$$
a\left|K_{1}+K_{2}\right|^{m}+a\left|K_{1}-K_{2}\right|^{m}+(2-a)\left|2 K_{2}\right|^{m}=2 \bar{\sigma}^{m}
$$


with

$$
\begin{aligned}
& K_{1}=\left(\sigma_{x x}+h \sigma_{y y}\right) / 2, \\
& K_{2}=\left\{\left[\left(\sigma_{x x}-h \sigma_{y y}\right) / 2\right]^{2}+\left[p \sigma_{x y}\right]^{2}\right\}^{0.5},
\end{aligned}
$$

where $a, h, p$ and $m$ are material constants. Non-quadratic yield functions have also been proposed by Chakrabarty [10] and others, but they will not be listed here.

It was pointed out by Hill [2] that none of the above yield functions accounts for the behavior that the tensile yield is the same along both the rolling and transverse directions, while the associated strain ratios are markedly different. Hill then proposed the following non-quadratic yield function in the same paper to account for this effect:

$$
\sigma_{1}^{2}-\left(2-\frac{\sigma_{\mathrm{u}}^{2}}{\sigma_{\mathrm{B}}^{2}}\right) \sigma_{1} \sigma_{2}+\sigma_{2}^{2}+\left\{(p+q)-\frac{\left(p \sigma_{1}+q \sigma_{2}\right)}{\sigma_{\mathrm{B}}}\right\} \sigma_{1} \sigma_{2}=\sigma_{\mathrm{u}}^{2} .
$$

This function was proposed with applications to thin sheet in mind and is restricted to the tension quadrant of the $\left(\sigma_{1}, \sigma_{2}\right)$ plane. An additional restriction of this form is that the tensile yield stress, denoted by $\sigma_{\mathrm{u}}$, is the same in rolling and transverse directions. The non-dimensional parameters $p$ and $q$ are positive or negative. The application of Eq. (8) is explained in Ref. [11], but that application was made in connection with the work contours which are not the same as the yield loci. Eq. (8) accounts for the anomaly. A non-quadratic yield function was also presented in Hill [2] for the case when tensile yield stresses in rolling and transverse directions are distinct.

The aforementioned yield criteria are phenomenological criteria. A polycrystal model based on Taylor [12] and Bishop and Hill [13], the TBH model, can also be used to describe the deformation of sheet metals. Such study has been reported by Barlat and Richmond [14] and Lege et al. [15]. By use of the TBH model the effects of rolling textures (Brass, Copper, S) and recrystallization textures (Cube, Goss) can be individually studied. The textures change with deformation, however, and the description of plastic deformation by the TBH model that accounts for the evolution of textures has not yet been demonstrated.

\subsection{Determination of coefficients of yield function and the $R$ ratio}

The coefficients of the yield function are determined from experiments. Several tests have been used in the literature for this purpose. They range from tests related to the determination of yield stresses to those that determine the width-to-thickness plastic strain ratio $R$. The latter tests have relatively poor accuracy however, and they involve assumptions and other factors to be further discussed. If the yield stresses are determined from experiments, tension test, biaxial tension test, bulge test, or compression test are usually used. Different investigators have used different sets of tests. After the yield stresses are known, they are substituted into the yield function to determine the coefficients $F, G, H$ and $N$ of the function by considering different special cases.

Knowing the coefficients, the flow rule may be used to obtain the expressions for plastic strain rate components. Then, the $R$ ratio may be determined. The ratio $R_{\alpha}$ for a tension specimen cut at an angle $\alpha$ with respect to the $x$-direction is defined by

$$
R_{x}=\frac{\mathrm{d} \varepsilon_{y^{\prime}}^{\mathrm{p}}}{\mathrm{d} \varepsilon_{z^{\prime}}^{\mathrm{p}}}
$$


where $x^{\prime}$ is along the longitudinal direction of the specimen; $y^{\prime}$ is the transverse direction; and $z^{\prime}=z$. Note that $x^{\prime}$ is making an angle $\alpha$ with the $x$-direction. By use of coordinate transformation, Eq. (9) is transformed into

$$
R_{x}=-\frac{\mathrm{d} \varepsilon_{x}^{\mathrm{p}} \sin ^{2} \alpha+\mathrm{d} \varepsilon_{y}^{\mathrm{p}} \cos ^{2} \alpha-2 \mathrm{~d} \varepsilon_{x y}^{\mathrm{p}} \sin \alpha \cos \alpha}{\mathrm{d} \varepsilon_{x}^{p}+\mathrm{d} \varepsilon_{y}^{\mathrm{p}}}
$$

where $\mathrm{d} \varepsilon_{x}^{\mathrm{p}}, \mathrm{d} \varepsilon_{y}^{\mathrm{p}}$ and $\mathrm{d} \varepsilon_{x y}^{\mathrm{p}}$ are components of the plastic strain increment. With these components determined from the flow rule, Eq. (10) is used to calculate $R_{\alpha}$ for different $\alpha$ angles. Usually, the theoretical $R_{\alpha}$-values do not compare favorably with those determined from experiment, but the agreement can be improved by considering non-quadratic yield functions.

The experimentally determined $R$ ratios have also been used to determine the coefficients of the yield function. Following [6], the $R$ ratios are related to the coefficients by

$$
1+2 R_{\alpha}=\frac{\sigma_{\mathbf{B}}^{m} / \tau^{m}-a \cos 2 \alpha+\{(m+2) / 2 m\} b \cos ^{2} 2 \alpha}{1-a \cos 2 \alpha+\{(m-2) / 2 m\} b \cos ^{2} 2 \alpha}
$$

It may be shown that

$$
\begin{aligned}
& \left(\sigma_{\mathrm{B}} / \tau\right)^{m}=1+2 R_{45} \\
& a=\frac{\left(R_{0}-R_{90}\right)\left[1-((m-2) / 2) R_{45}\right]}{\left(R_{0}+R_{90}\right)-(m-2) R_{0} R_{90}}, \\
& b=\frac{m\left[2 R_{0} R_{90}-R_{45}\left(R_{0}+R_{90}\right)\right]}{\left(R_{0}+R_{90}\right)-(m-2) R_{0} R_{90}}
\end{aligned}
$$

Thus, $a$ and $b$ may be determined if $R_{0}, R_{45}$ and $R_{90}$ are known. These are the $R$ ratios evaluated at $\alpha=0,45$ and $90^{\circ}$, respectively. $\left(\sigma_{\mathrm{B}} / \tau\right)^{m}, a$ and $b$ are the coefficients of the yield function given by Eqs. (3) and (4). Using Eq. (12a), $m$ can be determined if $\sigma_{\mathrm{B}}$ and $\tau$ are also known.

The above expressions suggest a modified method for the determination of the coefficients of the yield function. In this method, experimental data both for the $R$ ratios and the yield stresses are used to determine the coefficients $a, b$ and $m$, and the agreement between the theory and experiments are generally improved.

The coefficients $a, b$ and $m$ determined from the previous paragraph are generally not compatible with coefficients $F, G, H$ and $N$ found from tests to determine the yield stresses. These coefficients do not satisfy Eq. (4). The reason for the incompatibility is that they have been determined based on two different sets of experimental data, one set includes the $R$ ratios and the other does not. If both methods for the determination of the coefficients were correct, then Eq. (4) must be satisfied. This leads to the question of which method is correct. The determination of the coefficients will be discussed in a subsequent section.

\subsection{Some remarks}

Although several different yield functions have been discussed in Sections 2.1 and 2.2, they are yield functions of the same anisotropic theory of plasticity. This anisotropic theory proposes a yield 
function, assumes the normality condition for the plastic strain increments, and considers isotropic hardening in the tension quadrant of the two- or three-dimensional principal stress space. This anisotropic theory of plasticity is very different from the classical flow theory of plasticity. It is different in many aspects as follows: First, instead of a quadratic (initial) yield function for all metallic materials, the anisotropic theory uses a non-quadratic yield function with coefficients depending on the material being considered. Furthermore, instead of the usual isotropic-kinematic hardening concept, the anisotropic theory is concerned about the "anomalous behavior". Another discrepancy is related to the experimental determination of the coefficients of the yield function. Traditionally, the yield function has been determined independent of the flow rule. However, in this anisotropic theory, the flow rule has been used extensively in the determination of the coefficients of the yield function. The plastic strain ratios $R$ are expressed in terms of the flow rule, Eq. (10), and there are theoretical works, for example Eqs. (12), that express the coefficients of the yield function in terms of the plastic strain ratios, and, therefore, in terms of the flow rule.

These observations are interesting in the sense that the sheet metal would require a different theory of plasticity (the anisotropic theory) than the same material in other product forms. Since the sheet metal is orthotropic, an anisotropic yield function is reasonable. However, the aforementioned three discrepancies are too much a deviation that one wonders whether the two theories can be unified so that a simplified theory can be obtained for anisotropic materials.

\section{An extension of Hill's 1948 anisotropic theory}

It is proposed that a unified theory be formulated based on Eq. (1). In this case the yield function for sheet metals can be written as

$$
(G+H)\left(\sigma_{x}-r_{x}\right)^{2}-2 H\left(\sigma_{x}-r_{x}\right)\left(\sigma_{y}-r_{y}\right)+(H+F)\left(\sigma_{y}-r_{y}\right)^{2}+2 N\left(\sigma_{x y}-r_{x y}\right)^{2}=f^{2},
$$

where $r_{x}, r_{y}$ and $r_{x y}$, components of the back stress which are dependent on the deformation history, specify the center of the yield surface and are directly related to the kinematic hardening of the material; $f$ specifies the size of the yield surface and is an expression of isotropic hardening. In the case of a material without isotropic hardening, $f=1$. While Eq. (13) is valid for sheet metals, it can be generalized to obtain an expression for the three-dimensional anisotropic solid. It is assumed that the normality rule is valid so that the increments of plastic strain components are

$$
\begin{aligned}
& \mathrm{d} \varepsilon_{x}^{\mathrm{p}}=\mathrm{d} \lambda\left[(G+H)\left(\sigma_{x}-r_{x}\right)-H\left(\sigma_{y}-r_{y}\right)\right], \\
& \mathrm{d} \varepsilon_{y}^{\mathrm{p}}=\mathrm{d} \lambda\left[-H\left(\sigma_{x}-r_{x}\right)+(H+F)\left(\sigma_{y}-r_{y}\right)\right], \\
& \mathrm{d} \varepsilon_{x y}^{\mathrm{p}}=\mathrm{d} \lambda N\left(\sigma_{x y}-r_{x y}\right),
\end{aligned}
$$

where $\mathrm{d} \lambda$ is a proportional factor which varies with loading.

The coefficients of Eq. (13) may be determined at the condition of initial yielding. Thus, $r_{x}=r_{y}=r_{x y}=0$ and $f=1$, and Eq. (13) reduces to

$$
(G+H) \sigma_{x}^{2}-2 H \sigma_{x} \sigma_{y}+(H+F) \sigma_{y}^{2}+2 N \sigma_{x y}^{2}=1 .
$$


Note that $x$ denotes the rolling direction and $y$ the transverse direction. In a tension test along the $x$-direction, the stresses are $\sigma_{x} \neq 0, \sigma_{y}=\sigma_{x y}=0$, and Eq. (15) reduces to

$$
G+H=\left(\sigma_{x}^{\mathrm{Y}}\right)^{-2},
$$

where $\sigma_{x}^{\mathrm{Y}}$ is the yield stress in the $x$-direction. Similarly, a tension test along the $y$-direction leads to

$$
H+F=\left(\sigma_{y}^{Y}\right)^{-2},
$$

where $\sigma_{y}^{\mathrm{Y}}$ is the yield stress in the $y$-direction. Yielding under equibiaxial tension occurs when $\sigma_{x}=\sigma_{y}=\sigma_{\mathrm{B}}^{\mathrm{Y}}$. In this case, Eq. (15) reduces to

$$
G+F=\left(\sigma_{\mathrm{B}}^{Y}\right)^{-2}
$$

Due to the usual assumption that hydrostatic stress does not affect yielding, this is also the compressive yield stress perpendicular to the sheet. The through-thickness compression test was carried out by Naruse et al. [16]. Cylindrical specimens were prepared from discs of each sheet material glued together with an epoxy adhesive. The specimens were tested in compression using Teflon sheet and graphite grease for lubrication between the specimen ends and platen of the test machine. Factors of uncertainty arose from the epoxy adhesive, the Teflon sheet and graphite grease. Therefore, the equibiaxial tension test is preferred over the aforementioned compression test.

Eqs. (16a-c) can be solved for $G, F$, and $H$ as

$$
\begin{aligned}
& 2 G=\frac{1}{\left(\sigma_{x}^{\mathrm{Y}}\right)^{2}}-\frac{1}{\left(\sigma_{y}^{\mathrm{Y}}\right)^{2}}+\frac{1}{\left(\sigma_{\mathrm{B}}^{\mathrm{Y}}\right)^{2}}, \\
& 2 F=\frac{1}{\left(\sigma_{y}^{\mathrm{Y}}\right)^{2}}-\frac{1}{\left(\sigma_{x}^{\mathrm{Y}}\right)^{2}}+\frac{1}{\left(\sigma_{\mathrm{B}}^{\mathrm{Y}}\right)^{2}}, \\
& 2 H=\frac{1}{\left(\sigma_{x}^{\mathrm{Y}}\right)^{2}}+\frac{1}{\left(\sigma_{y}^{\mathrm{Y}}\right)^{2}}-\frac{1}{\left(\sigma_{\mathrm{B}}^{\mathrm{Y}}\right)^{2}} .
\end{aligned}
$$

Finally, the coefficient $N$ may be determined from the tension test of a specimen cut at $45^{\circ}$ angle with the $x$-direction. The tensile yield stress for this specimen is denoted by $\sigma_{45}$, and $\sigma_{x}=\sigma_{y}=$ $\sigma_{x y}=\frac{1}{2} \sigma_{45}$. Using this condition, it may be found from Eq. (15) that

$$
2 N=\left(\frac{\sigma_{45}}{2}\right)^{-2}-\left(\sigma_{\mathrm{B}}^{\mathrm{Y}}\right)^{-2}
$$

Since $\sigma_{45}$ is easily determined experimentally, $N$ is thus determined from Eq. (17d). The shear yield stress $\sigma_{x y}^{\mathrm{Y}}$ is determined in pure shear with material element parallel to the orthotropic axes. It may be shown from Eq. (15) that

$$
\sigma_{x y}^{\mathrm{Y}}=\frac{1}{\sqrt{2 N}} .
$$

In sheet metals, however, pure shear is difficult to realize experimentally. Also, the simple shear test is sometimes used in the literature to determine $N$. The stress state of this test is never simple, 
however, and the test can at best be used as an approximation. It has thus been shown that the coefficients of the yield function can be determined by a set of tension tests. Well-controlled tension tests are simple to perform. The equibiaxial test can be carried out by use of cruciform specimens as in $\operatorname{Refs}[17,18]$.

With the coefficients determined, Eqs. (13) and (14) can be applied to solve problems of sheet metals. The challenge is then to correctly determine $r_{x}, r_{y}, r_{x y}$ and $f$. The back stress components $r_{x}, r_{y}$ and $r_{x y}$ may be correlated with the evolution of rolling textures in the sheet. In this way, the rolled sheet at different stages of rolling, i.e. at different percentages of thickness reduction, may be studied and will be discussed in a later section. In the case of as-received materials, some materials may show anomalous behavior at initial yielding due to initial anisotropy, then for this as-received material the initial $r_{x}, r_{y}$, and $r_{x y}$ are not zero. However, the anomalous behavior for some as-received materials may not be apparent, then $r_{x}, r_{y}$, and $r_{x y}$ may be put to zero as an approximation.

Since the plastic strain ratio $R_{\alpha}$ is an important parameter in sheet metal forming, Eqs. (13) and (14) will now be applied to investigate $R_{\alpha}$. In order to obtain a theoretical description of this parameter, the test condition that is used to determine $R_{x}$ must be closely followed. There are many factors of uncertainty associated with the plastic strain ratio test. Experiments show that the $R$-ratio varies with strain. Thus, the $R$-ratio depends on the strain hardening of the material. According to Mellor [19] for titanium 115, the $R$-ratio varies greatly with the increasing plastic strain if it is defined as the ratio of the width to thickness strain. The ratio varies the most at the transition zone from the elastic to plastic strain. But if the $R$-ratio is defined as the ratio of plastic strain increments, then it almost remains constant over the whole plastic strain range tested, up to a strain of $13 \%$. The importance of elastic strain in the definition of the $R$-ratio is well demonstrated by the experiments. However, the elastic strain is often neglected in the study of metal sheet. The experimental results are not sufficiently accurate to allow computation of the ratio of plastic strain increments closer to the initial yielding zone. The curve for $R$ rises slightly with decreasing strain, but an extrapolation of this curve to zero strain is not recommended. Experiments were also conducted by Lin and Ding [18] for as received and cold-rolled aluminum sheets using cruciform plate specimen. A similar conclusion was also obtained regarding the $R$-ratio at small plastic strain. The authors stated that $R$ could not be reasonably determined when the plastic strain is infinitesimal due to the quite severe scattering of the plastic strain increments. Hill et al. [11] tested thin-walled brass tubes and assessed $R_{0}$ and $R_{90}$ in the post-yield range along proportional strain paths. The values of $R_{0}$ and $R_{90}$ at the initial yielding are ill-defined however. The axial strain and the change of diameter were carefully measured, but no measurements of the thickness strain were reported. These additional information has not been considered in most theoretical work which uses the $R$-ratio as a parameter to define other properties of the sheet metal. It is the opinion of the authors that additional investigation of the $R$-ratio is warranted both theoretically and experimentally. Particularly, the $R$ value must be determined at the same axial strain level as the one used to determine the yield stress.

For a tensile specimen at an angle $\alpha$ to the rolling direction,

$$
\sigma_{x}=s \cos ^{2} \alpha, \quad \sigma_{y}=s \sin ^{2} \alpha \quad \text { and } \quad \sigma_{x y}=s \sin \alpha \cos \alpha,
$$

where $s$ is the tensile stress applied to the specimen. 
Substituting Eqs. (14) and (19) into Eq. (10), the $R$-ratio is obtained as

$$
\begin{aligned}
R_{\alpha}= & -\left\{\left[(G+H)\left(s \cos ^{2} \alpha-r_{x}\right)-H\left(\sin ^{2} \alpha-r_{y}\right)\right] \sin ^{2} \alpha\right. \\
& +\left[(H+F)\left(s \sin ^{2} \alpha-r_{y}\right)-H\left(s \cos ^{2} \alpha-r_{x}\right)\right] \cos ^{2} \alpha \\
& \left.-2 N\left(s \sin \alpha \cos \alpha-r_{x y}\right) \sin \alpha \cos \alpha\right\} /\left\{G\left(s \cos ^{2} \alpha-r_{x}\right)+F\left(\sin ^{2} \alpha-r_{y}\right)\right\} .
\end{aligned}
$$

Note that the isotropic hardening parameter $f$ does not appear in Eq. (20). From this equation, the following expressions can be found:

$$
\begin{aligned}
& R_{0}=\frac{s H-H r_{x}+(H+F) r_{y}}{s G-G r_{x}-F r_{y}} \\
& R_{90}=\frac{s H+(G+H) r_{x}-H r_{y}}{s F-G r_{x}-F r_{y}} \\
& R_{45}=\frac{1}{2}\left[\frac{2 N\left(s-2 r_{x y}\right)}{s(G+F)-2 G r_{x}-2 F r_{y}}-1\right] .
\end{aligned}
$$

When $r_{x}=r_{y}=r_{x y}=0$, Eqs. (21) reduce to

$$
\begin{aligned}
& R_{0}=\frac{H}{G}, \\
& R_{90}=\frac{H}{F}, \\
& R_{45}=\frac{1}{2}\left(\frac{2 N}{G+F}-1\right),
\end{aligned}
$$

which is a well-known result.

\section{Anisotropic sheet with thickness reduction by further rolling}

Eqs. (22), although in the same form as those obtained by Hill [6], do not describe the experimental $R$-ratios if the coefficients of the quadratic yield function are determined by the yield stresses as suggested in the previous section. To obtain a correct determination of the $R$-ratios, the experimental procedure that is used to determine the $R$-ratios must be closely followed. During this procedure, the material has been stretched and, therefore, strain hardened. Thus, it is important to account for the effect of strain hardening in the theoretical determination of the $R$-ratios.

The strain hardening can occur in an isotropic or in a kinematic mode. It can also have a combined isotropic-kinematic hardening, depending on the material under consideration. Strain hardening occurs in two stages. The first-stage strain hardening is developed during the metalforming process. For instance, the annealed metal sheet can be considered as in the state of no strain hardening. When the thickness of a metal sheet is reduced by further rolling, the strain 
hardening will develop and this strain hardening can play an important role and should be accounted for in the theoretical work. The second-stage strain hardening develops when tension specimen is cut from the metal sheet and tested in tension. The effect of strain hardening has not been sufficiently studied in the literature of sheet metals.

The first-stage strain hardening can be determined by considering the initial yield locus in the principal stress space. For the annealed metal sheet, Eq. (15) is fitted to the experimental yield locus. On the other hand, Eq. (13) can be fitted to the yield locus determined from a metal sheet with a specified thickness reduction. By comparing the two loci, the displacement of the center of the yield locus and the expansion of the size of the yield locus can be determined. These correspond, respectively, to the first-stage kinematic hardening $\left(r_{x}^{0}, r_{y}^{0}, r_{x y}^{0}\right)$ and isotropic hardening $f^{0}$. The superscript 0 denotes the first-stage strain hardening. Generally, the principal directions for the stress $\sigma_{i j}$ and the back stress $r_{i j}$ do not coincide.

For sheet metals, experimental yield locus can only be determined in the first quadrant. Since it is not practical to apply compressive load to the sheet, a complete two-dimensional yield locus is not available. The incomplete yield locus is subjected to different interpretations. One popular interpretation is to consider the "anomalous behavior", but it leads to the undesirable high-order yield function. It is undesirable in that it is difficult to apply; the convexity of the yield function needs to be investigated; and, from a unified viewpoint, the non-quadratic yield function will have to reduce to the quadratic function when the material is initially isotropic and gradually becomes anisotropic due to deformation. A different viewpoint is taken by the present authors that the yield locus has undergone isotropic as well as kinematic hardening. When the kinematic hardening is considered, the experimental points can be fitted by the quadratic yield function, Eq. (13). Therefore, the "anomalous behavior" is but a matter of choice of interpretation.

The second-stage strain hardening develops when tensile specimens, which were cut from the metal sheet at various angles, are pulled in uniaxial tension. During the tension test, additional strain hardening occurs and they are denoted by $\left(\bar{r}_{x}, \bar{r}_{y}, \bar{r}_{x y} \neq 0\right)$ for kinematic hardening and $\bar{f}$ for isotropic hardening. Thus, the components of the back stress are

$$
\begin{aligned}
& r_{x}=r_{x}^{0}+\bar{r}_{x}, \\
& r_{y}=r_{y}^{0}+\bar{r}_{y}, \\
& r_{x y}=r_{x y}^{0}+\bar{r}_{x y} .
\end{aligned}
$$

Introduce now the principal direction of the back stress denoted by $\left(\theta^{0}, \bar{\theta}\right)$, measured counterclockwise from the $x$-axis, and the principal components denoted by $\left(r_{\max }^{0}, r_{\min }^{0}\right)$ and $\left(\bar{r}_{\max }, \bar{r}_{\min }\right)$. The pressions are

$$
\begin{aligned}
& \theta^{0}=\frac{1}{2} \tan ^{-1} \frac{2 r_{x y}^{0}}{r_{x}^{0}-r_{y}^{0}}, \\
& r_{\max }^{0}=r_{x}^{0} \cos ^{2} \theta^{0}+r_{y}^{0} \sin ^{2} \theta^{0}+2 r_{x y}^{0} \sin \theta^{0} \cos \theta^{0}, \\
& r_{\min }^{0}=r_{x}^{0} \sin ^{2} \theta^{0}+r_{y}^{0} \cos ^{2} \theta^{0}-2 r_{x y}^{0} \sin \theta^{0} \cos \theta^{0},
\end{aligned}
$$




$$
\begin{aligned}
& \bar{\theta}=\frac{1}{2} \tan ^{-1} \frac{2 \bar{r}_{x y}}{\bar{r}_{x}-\bar{r}_{y}}, \\
& \bar{r}_{\max }=\bar{r}_{x} \cos ^{2} \bar{\theta}+\bar{r}_{y} \sin ^{2} \bar{\theta}+2 \bar{r}_{x y} \sin \bar{\theta} \cos \bar{\theta}, \\
& \bar{r}_{\min }=\bar{r}_{x} \sin ^{2} \bar{\theta}+\bar{r}_{y} \cos ^{2} \bar{\theta}-2 \bar{r}_{x y} \sin \bar{\theta} \cos \bar{\theta} .
\end{aligned}
$$

If the coefficients $F, G, H$ and $N$ are known and $\left(\theta^{0}, r_{\max }^{0}, r_{\min }^{0}\right)$ and $\left(\bar{\theta}, \bar{r}_{\max }, \bar{r}_{\min }\right)$ are specified, which will be further discussed in the next section, then Eqs. (23)-(25) may be used to determine $r_{x}, r_{y}$ and $r_{x y}$. Thus, Eq. (20) may be used to find the plastic strain ratio $R_{\alpha}$ at any angle $\alpha$.

When the initial back stress is not zero, which corresponds to the metal sheet with a given amount of thickness reduction due to rolling, the tensile yield stress of a specimen cut at angle $\alpha$ with respect to the rolling direction is determined by use of the yield function (13). If $\sigma_{x}$ denotes the tensile yield stress for the tension specimen, then $\sigma_{\alpha}$ is related to the stress components by

$$
\sigma_{x}=\sigma_{x} \cos ^{2} \alpha, \quad \sigma_{y}=\sigma_{x} \sin ^{2} \alpha \text { and } \sigma_{x y}=\sigma_{x} \sin \alpha \cos \alpha .
$$

Upon the substitution of Eq. (26), Eq. (13) becomes

$$
\begin{aligned}
& \left(\sigma_{\alpha}\right)^{2}\left[(G+H) \cos ^{4} \alpha-2 H \cos ^{2} \alpha \sin ^{2} \alpha+(F+H) \sin ^{4} \alpha+2 N \sin ^{2} \alpha \cos ^{2} \alpha\right] \\
& \quad+\sigma_{x}\left[-2(G+H) r_{x} \cos ^{2} \alpha+2 H\left(r_{x} \sin ^{2} \alpha+r_{y} \cos ^{2} \alpha\right)\right. \\
& \left.\quad-2(H+F) r_{y} \sin ^{2} \alpha-4 N r_{x y} \sin \alpha \cos \alpha\right]+\hat{r}=f^{2} .
\end{aligned}
$$

This is an algebraic quadratic equation in $\sigma_{\alpha}$ where all coefficients of the equation are known. $\hat{r}$ in the above equation is given by

$$
\hat{r}=\left(\frac{r_{x}}{\sigma_{x}^{Y}}\right)^{2}-2 H r_{x} r_{y}+\left(\frac{r_{y}}{\sigma_{y}^{Y}}\right)^{2}+2 N\left(r_{x y}\right)^{2}
$$

Thus, for a given $\alpha$, the tensile yield stress $\sigma_{x}$ at the on-set of second stage yielding may be determined from Eq. (27) by setting $\bar{r}_{x}=\bar{r}_{y}=\bar{r}_{x y}=0$. Therefore, from Eq. (23), $r_{x}=r_{x}^{0}, r_{y}=r_{y}^{0}$, $r_{x y}=r_{x y}^{0}, f=f^{0}$, and from Eq. (27), the following special cases are obtained:

$$
\begin{aligned}
& \sigma_{0}^{2}(G+H)+\sigma_{0}\left[-2(G+H) r_{x}^{0}+2 H r_{y}^{0}\right]+\hat{r}^{0}=\left(f^{0}\right)^{2}, \\
& \sigma_{90}^{2}(H+F)+\sigma_{90}\left[2 H r_{x}^{0}-2(H+F) r_{y}^{0}\right]+\hat{r}^{0}=\left(f^{0}\right)^{2}, \\
& \left(\frac{\sigma_{45}}{2}\right)^{2}(G+F+2 N)-\sigma_{45}\left[G r_{x}^{0}+F r_{y}^{0}+2 N r_{x y}^{0}\right]+\hat{r}^{0}=\left(f^{0}\right)^{2} .
\end{aligned}
$$

In Eq. (29), $\hat{r}^{0}$ denotes the value of $\hat{r}$ when $r_{x}=r_{x}^{0}, r_{y}=r_{y}^{0}$, and $r_{x y}=r_{x y}^{0}$. Eq. (27) may also be plotted to obtain the variation of $\sigma_{\alpha}$ with respect to $\alpha$. When the sheet is at the as-received condition, in many cases, it may be set $r_{x}^{0}=r_{y}^{0}=r_{x y}^{0}=0$ and $f^{0}=1$. Then, using Eqs. (16) and (17), Eqs. (29) reduce to

$$
\sigma_{0}=\sigma_{x}^{\mathrm{Y}}, \quad \sigma_{90}=\sigma_{y}^{\mathrm{Y}}, \quad \text { and } \quad \sigma_{45}=\frac{2}{\sqrt{1 /\left(\sigma_{x y}^{\mathrm{Y}}\right)^{2}+1 /\left(\sigma_{\mathrm{B}}^{\mathrm{Y}}\right)^{2}}}
$$

Note that Eqs. (30) are the same as those of Hill [6]. 


\section{Applications}

Equations derived in Section 4 are now applied to describe the plastic behavior of metal sheets. Theoretical results are compared with experimental results. The first experimental results considered herein are those obtained by Lin and Ding [18] for as-received and cold-rolled aluminum sheets of 25 and $50 \%$ thickness reductions, respectively. Note that this is a case of $R<1$. Fig. 1(a) shows the yield loci determined from the quadratic yield function (13) compared with experimental
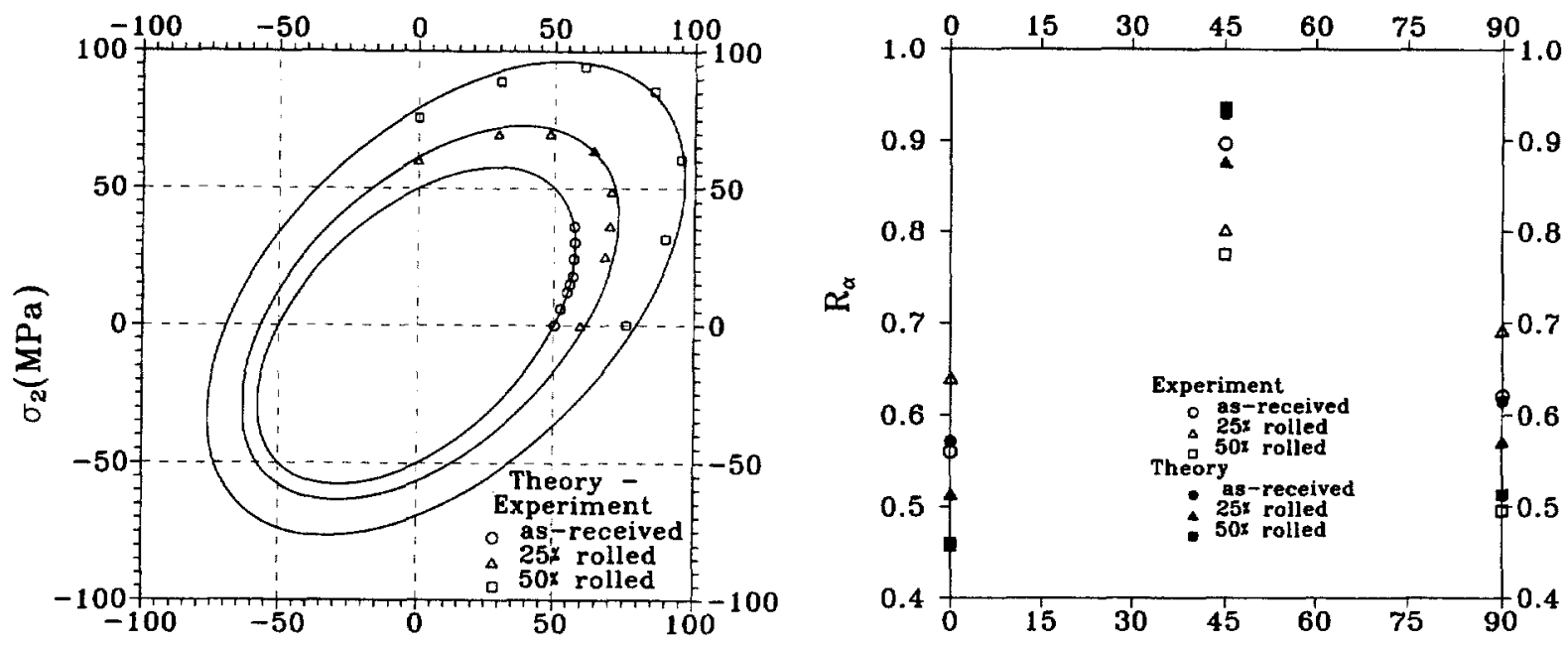

(a)

$$
\sigma_{1}(\mathrm{MPa})
$$

(b)

$\alpha$ (degrees)

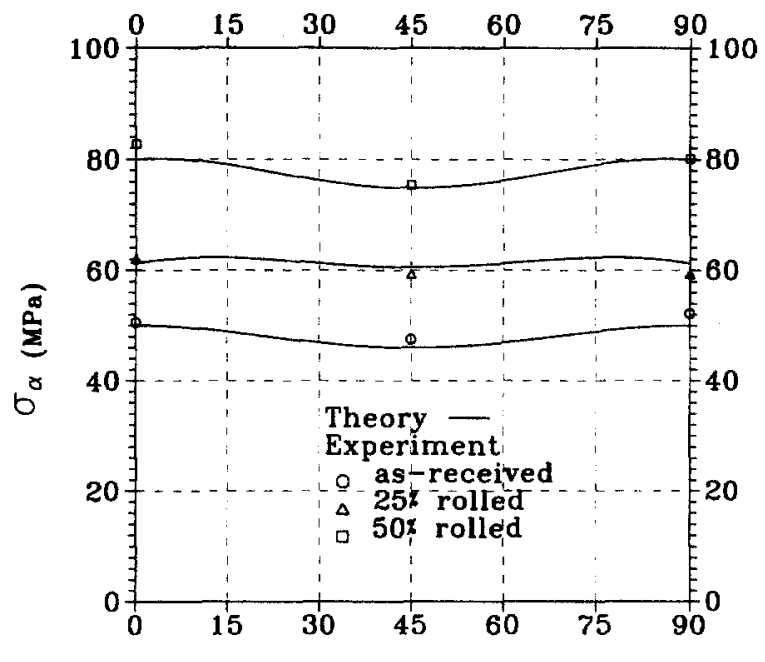

(c)

$\alpha$ (degrees)

Fig. 1. (a) Yield loci for $1100-\mathrm{F}$ aluminum sheet. (b) Plastic strain ratio, $R_{\alpha}$, for $1100-\mathrm{F}$ aluminum sheet. (c) Yield stress for tension specimen, $\sigma_{x}$, for $1100-\mathrm{F}$ aluminum sheet. 
data. The yield locus for the as-received condition are determined using $r_{x}^{0}=r_{y}^{0}=r_{x y}^{0}=0 \mathrm{MPa}$, $f^{0}=1, \sigma_{x}^{\mathrm{Y}}=\sigma_{y}^{\mathrm{Y}}=\sigma_{\mathrm{B}}^{\mathrm{Y}}=50 \mathrm{MPa}$; for the sheet with $25 \%$ thickness reduction, using $r_{x}^{0}(25 \%)=$ $r_{y}^{0}(25 \%)=4.7 \mathrm{MPa}, f^{0}(25 \%)=1.185$; and, for the sheet with $50 \%$ thickness reduction, using $r_{x}^{0}(50 \%)=r_{y}^{0}(50 \%)=10.1 \mathrm{MPa}, f^{0}(50 \%)=1.509$. Note that the first-stage back stress and the isotropic hardening function are functions of the strain. Since the Lin-Ding data are given only in the first quadrant of the principal stress space, it opens up various possibilities for describing the data. One could consider the behavior of anomaly as most researchers do; or one could consider the kinematic hardening behavior as proposed in this investigation. It is shown here that the experimental data can be reasonably described by use of the quadratic yield criterion, given by Eq. (13), without the consideration of the "anomalous behavior". The aforementioned values for $r_{x}^{0}$, $r_{y}^{0}$ and $f^{0}$ were determined by an optimization procedure. No uniqueness for these values is claimed.

The plastic strain ratio $R_{\alpha}$ for a tension specimen cut at an angle $\alpha$ is determined from Eqs. (20) and (21). The parameters of the model and results for all cases are listed in Table 1 . The results are also plotted in Fig. 1(b) together with the experimental data of Lin and Ding [18]. The knowledge of principal direction and values $\left(\theta^{0}, r_{\max }^{0}, r_{\min }^{0}\right)$ is equivalent to the knowledge of $\left(r_{x}^{0}, r_{y}^{0}, r_{x y}^{0}\right)$. In the kinematic hardening of the yield locus, only $r_{x}^{0}$ and $r_{y}^{0}$ can be determined as in Fig. 1(a). Since the principal directions of stress tensor and back stress do not usually coincide, the component $r_{x y}^{0} \neq 0$. But, the value of this component has not been determined experimentally. Thus, the values of $\theta^{0}$, $r_{\max }^{0}$ and $r_{\min }^{0}$ are not determined by the available data. In addition, during the tension test on specimen cut at an angle $\alpha$, necessary measurements have not been taken that would benefit a careful theoretical study of the plastic behavior of the tension test. Therefore, information about $\left(\bar{r}_{x}, \bar{r}_{y}, \bar{r}_{x y}\right)$, or equivalently $\left(\bar{\theta}, \bar{r}_{\max }, \bar{r}_{\min }\right)$, is not available.

Due to the lack of experimental data, it is shown here that, by use of equations of Section 4 , a heuristic choice of the kinematic hardening values does lead to a reasonable description of experimental data for the yield locus, the strain ratio $R_{x}$, and the yield stress $\sigma_{\alpha}$. The criteria for the determination of the kinematic hardening values $\left(\bar{\theta}, \bar{r}_{\max }, \bar{r}_{\min }\right)$ are: (a) the calculated $R_{\alpha}$ values for all $\alpha$ should fit the experimental data; (b) the motion of the center of yield locus should be smooth as $\alpha$ varies; and (c) the kinematic hardening values used in the calculation should be able to capture the essential features of the tension test. A commercially available optimization computer program is used to cope with criterion (a). Criterion (b) is translated into the requirement that the $\bar{\theta}$, $\bar{r}_{\max }$ and $\bar{r}_{\min }$ versus $\alpha$ curves be smooth. Criterion (c) is further explained and $\left(\bar{r}_{x}, \bar{r}_{y}, \bar{r}_{x y}\right)$ is used to aid the discussion. Note that the knowledge of $\left(\bar{r}_{x}, \bar{r}_{y}, \bar{r}_{x y}\right)$ is equivalent to the knowledge of $\left(\bar{\theta}, \bar{r}_{\max }\right.$, $\left.\bar{r}_{\min }\right)$ as previously mentioned. Prager's linear kinematic hardening rule [20] is used as an approximation. According to this rule, the motion of center of yield locus is proportional to the plastic strain rate vector, which is normal to the yield locus. Thus, for the two-dimensional principal stress space under consideration, the case of $\alpha=0^{\circ}$ corresponds to $\bar{r}_{x}>0, \bar{r}_{y}<0$ and $\bar{r}_{x y} \neq 0$; the case of $\alpha=45^{\circ}$ leads to $\bar{r}_{x}=\bar{r}_{y^{\prime}}>0$ and $\bar{r}_{x y} \neq 0$, if the yield locus is an ellipse, and $\bar{r}_{x} \neq \bar{r}_{y}>0$ and $\bar{r}_{x y} \neq 0$, if the yield locus is not an ellipse; and the case of $\alpha=90^{\circ}$ corresponds to $\bar{r}_{x}<0, \bar{r}_{y}>0$ and $\bar{r}_{x y} \neq 0$.

The aforementioned criteria are used in the determination of kinematic hardening values of Table 1 . Note that these are generally functions of the strain. But, numerical values are given in the table which correspond to the state of kinematic hardening when yield stress is being determined in the tension tests. The same criteria and method are used for Tables 2 and 3 which are related to 
Table 1

Plastic strain ratios $R_{\alpha}$ and yield stress $\sigma_{\alpha}($ Lin $\&$ Ding)

\begin{tabular}{|c|c|c|c|c|c|c|c|c|c|}
\hline \multicolumn{10}{|c|}{$\begin{array}{l}F=G=H=2 \times 10^{-4}(\mathrm{MPa})^{-2} \\
2 N=14.904 \times 10^{-4}(\mathrm{MPa})^{-2}\end{array}$} \\
\hline Condition & $x$ & $\theta^{\circ}$ & $r_{\max }^{0}$ & $r_{\min }^{0}$ & $\bar{\theta}$ & $\bar{r}_{\max }$ & $\bar{r}_{\min }$ & $R_{\alpha}$ & $\sigma_{\alpha}$ \\
\hline As-received & 0.0 & 0.0 & 0.0 & 0.0 & 26.84 & 9.30 & -11.80 & 0.571 & 50.0 \\
\hline As-received & 45.0 & 0.0 & 0.0 & 0.0 & 40.00 & 12.13 & -5.13 & 0.930 & 46.0 \\
\hline As-received & 90.0 & 0.0 & 0.0 & 0.0 & 109.50 & 19.00 & -8.01 & 0.615 & 50.0 \\
\hline C-W 25\% & 0.0 & 45.0 & 7.29 & 2.12 & 24.41 & 9.54 & -17.04 & 0.514 & 61.3 \\
\hline C-W $25 \%$ & 45.0 & 45.0 & 7.29 & 2.12 & 40.73 & 13.61 & -6.61 & 0.877 & 60.5 \\
\hline C-W $25 \%$ & 90.0 & 45.0 & 7.29 & 2.12 & 101.20 & 45.48 & -6.98 & 0.571 & 61.3 \\
\hline C-W $50 \%$ & 0.0 & 45.0 & 11.11 & 9.09 & 26.60 & 12.61 & -25.36 & 0.461 & 80.0 \\
\hline C-W $50 \%$ & 45.0 & 45.0 & 11.11 & 9.09 & 42.18 & 18.77 & -11.77 & 0.936 & 74.9 \\
\hline C-W $50 \%$ & 90.0 & 45.0 & 11.11 & 9.09 & 121.12 & 50.44 & -35.44 & 0.514 & 80.0 \\
\hline
\end{tabular}

Table 2

Plastic strain ratios $R_{\alpha}$ and yield stress $\sigma_{\alpha}$ for annealed aluminum sheet

\begin{tabular}{|c|c|c|c|c|c|c|c|c|c|}
\hline \multicolumn{10}{|c|}{$\begin{array}{l}F=1.535 \times 10^{-5}(\mathrm{MPa})^{-2} \\
G=1.029 \times 10^{-5}(\mathrm{MPa})^{-2} \\
H=1.711 \times 10^{-5}(\mathrm{MPa})^{-2} \\
2 N=11.408 \times 10^{-5}(\mathrm{MPa})^{-2} \\
\sigma_{x}=191 \mathrm{MPa} \\
\sigma_{b}=197.5 \mathrm{MPa}\end{array}$} \\
\hline Condition & $\alpha$ & $\theta^{\circ}$ & $r_{\max }^{0}$ & $r_{\min }^{0}$ & $\bar{\theta}$ & $\bar{r}_{\max }$ & $\bar{r}_{\min }$ & $R_{\alpha}$ & $\sigma_{b} / \sigma_{x}$ \\
\hline Annealed & 0.0 & 0.0 & 0.0 & 0.0 & 6.44 & 73.43 & -27.22 & 0.735 & 1.034 \\
\hline Annealed & 15.0 & 0.0 & 0.0 & 0.0 & 5.40 & 95.66 & -28.53 & 0.704 & 1.063 \\
\hline Annealed & 22.5 & 0.0 & 0.0 & 0.0 & 6.51 & 101.78 & -26.85 & 1.002 & 1.093 \\
\hline Annealed & 30.0 & 0.0 & 0.0 & 0.0 & 8.63 & 104.82 & -23.88 & 1.358 & 1.124 \\
\hline Annealed & 45.0 & 0.0 & 0.0 & 0.0 & 16.83 & 104.39 & -16.78 & 1.735 & 1.167 \\
\hline Annealed & 60.0 & 0.0 & 0.0 & 0.0 & 31.55 & 105.34 & -18.18 & 1.373 & 1.167 \\
\hline Annealed & 67.5 & 0.0 & 0.0 & 0.0 & 39.87 & 111.71 & -27.45 & 1.032 & 1.155 \\
\hline Annealed & 75.0 & 0.0 & 0.0 & 0.0 & 47.06 & 123.62 & -44.05 & 0.724 & 1.141 \\
\hline Annealed & 90.0 & 0.0 & 0.0 & 0.0 & 56.68 & 162.25 & -97.40 & 0.712 & 1.125 \\
\hline
\end{tabular}

other examples to be subsequently discussed. For the current example, it is seen from Fig. 1(b) that $R_{\alpha}$ varies with $\alpha$ and no definite trend of variation for $R_{\alpha}$ as a function of reduction of sheet thickness is found from the experimental data. Apparently further experimental investigation need be conducted. Only results for $\alpha=0,45$ and $90^{\circ}$ are shown in the figure, since $R_{\alpha}$ for other $\alpha$ 's have not been experimentally determined. Eqs. (27) and (29) are used to determine the yield stresses for the tension specimens. The results are shown in Fig. 1(c) and also listed in Table 1. It is seen from Fig. 1(c) that $\sigma_{x}$ increases with the amount of reduction of sheet thickness. 
Table 3

Plastic strain ratios $R_{\alpha}$ and yield stress $\sigma_{\alpha}$ for SPCE steel sheet

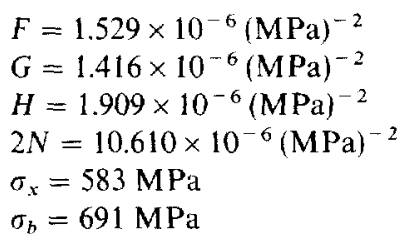

\begin{tabular}{lccccccccc} 
Condition & $\alpha$ & $\theta^{\circ}$ & $r_{\max }^{0}$ & $r_{\min }^{0}$ & $\bar{\theta}$ & $\bar{r}_{\max }$ & $\bar{r}_{\min }$ & $R_{\alpha}$ & $\sigma_{h / \sigma_{z}}$ \\
Deep-draw & 0.0 & 45.0 & 104.5 & 85.5 & 24.87 & 99.84 & -153.59 & 1.348 & 1.182 \\
Deep-draw & 15.0 & 45.0 & 104.5 & 85.5 & 30.31 & 142.11 & -128.64 & 1.264 & 1.167 \\
Deep-draw & 22.5 & 45.0 & 104.5 & 85.5 & 32.72 & 158.05 & -119.51 & 1.200 & 1.162 \\
Deep-draw & 30.0 & 45.0 & 104.5 & 85.5 & 35.02 & 170.37 & -112.46 & 1.146 & 1.159 \\
Deep-draw & 45.0 & 45.0 & 104.5 & 85.5 & 39.51 & 184.01 & -104.42 & 1.140 & 1.159 \\
Deep-draw & 60.0 & 45.0 & 104.5 & 85.5 & 44.21 & 183.23 & -104.75 & 1.295 & 1.166 \\
Deep-draw & 67.5 & 45.0 & 104.5 & 85.5 & 46.76 & 177.71 & -108.32 & 1.405 & 1.172 \\
Deep-draw & 75.0 & 45.0 & 104.5 & 85.5 & 49.51 & 169.03 & -114.44 & 1.512 & 1.180 \\
Deep-draw & 90.0 & 45.0 & 104.5 & 85.5 & 55.74 & 143.38 & -135.44 & 1.659 & 1.197 \\
\hline
\end{tabular}

It should be remarked that no predictions of the model are claimed. Predictions can be done only when experimental data for the back stress are available, or, in a theoretical work, a model for the evolution of the back stress is used. In this paper, it is merely shown that reasonable agreement with the experimental data can be achieved by use of the equations of Section 4 with heuristic choices of parameters.

The next application refers to experimental results of Naruse et al. [16]. Experimental data for yield locus, $R_{x}$, and $\sigma_{b} / \sigma_{x}$ are presented in the paper for annealed commercially pure aluminum sheets (A1100P) and deep-drawing-quality steel sheets (SPCE), where $\sigma_{\mathrm{b}}=\sigma_{\mathrm{B}}^{Y}$ is the equibiaxial yield stress used by Naruse et al. [16] to report their experimental data. Equation (15) is used to calculate the yield locus for annealed aluminum, i.e., $r_{x}^{0}=r_{y}^{0}=r_{x y}^{0}=0$ and $f^{0}=1$. From Table 1 of

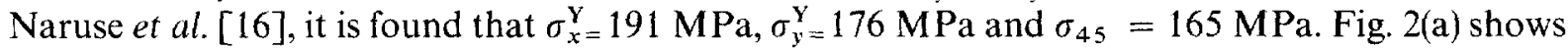
the yield locus together with the experimental data, in which the stress is normalized by its value at $x=0^{\circ}$. It is then determined that $\sigma_{\mathrm{B}}^{\mathrm{Y}}=197.5 \mathrm{MPa}$ (adjusted to account for kinematic hardening). The following coefficients are then determined: $F=1.535 \times 10^{-5}(\mathrm{MPa})^{-2}, G=1.029 \times 10^{-5}$ $(\mathrm{MPa})^{-2}, H=1.711 \times 10^{-5}(\mathrm{MPa})^{-2}$, and $2 N=11.408 \times 10^{-5}(\mathrm{MPa})^{-2}$. The parameters used to determine $R_{\alpha}$ and $\sigma_{x}$ are listed in Table 2 for $\alpha=0,15,22.5,30,45,60,67.5,75$ and $90^{\circ}$. The results for $R_{\alpha}$ and $\sigma_{b} / \sigma_{x}$ are also listed in the table and plotted in Fig. 2(b) and (c). Note that this is a case where $R$ can be less than, equal to, or greater than one depending on the angle $\alpha$. Fig. 3(a) shows the principal angle $\bar{\theta}$ as a function of $\alpha$ and Fig. $3(\mathrm{~b})$ shows the principal back stresses $\bar{r}_{\max }$ and $\bar{r}_{\min }$ as a function of $\alpha$. The criteria for the determination of these values are the same as those given for the Lin-Ding data.

Eq. (13) is used to calculate the yield locus for the SPCE steel sheet. It has been determined that the sheet has the initial back stress of $r_{x}^{0}=r_{y}^{0}=95.0 \mathrm{MPa}$ and $f^{0}=1$. The following values are used: $\sigma_{x}^{\mathrm{Y}}=583 \mathrm{MPa}, \sigma_{y}^{\mathrm{Y}}=561 \mathrm{MPa}, \sigma_{\mathrm{B}}^{\mathrm{Y}}=691 \mathrm{MPa}$ (adjusted to account for kinematic hardening) 

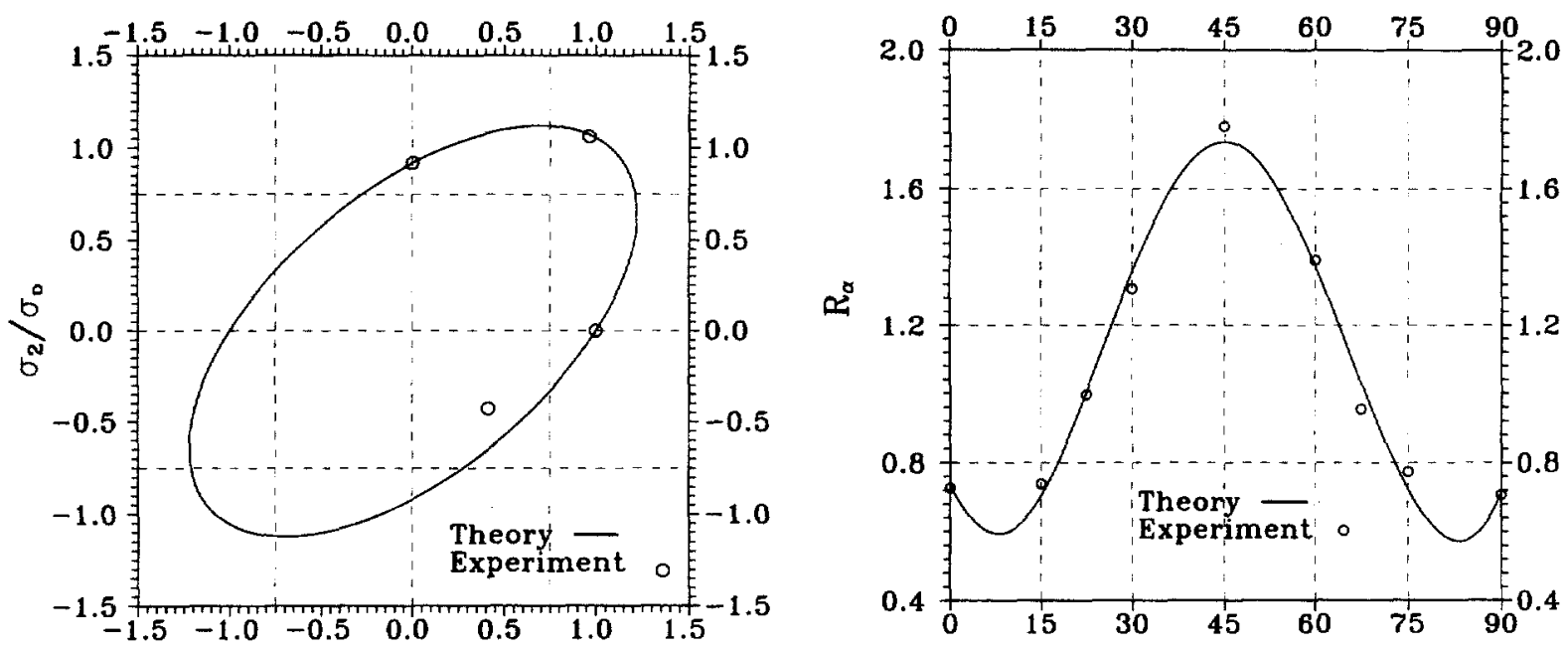

(a) $\sigma_{1} / \sigma_{0}$

(b)

$\alpha$ (degrees)

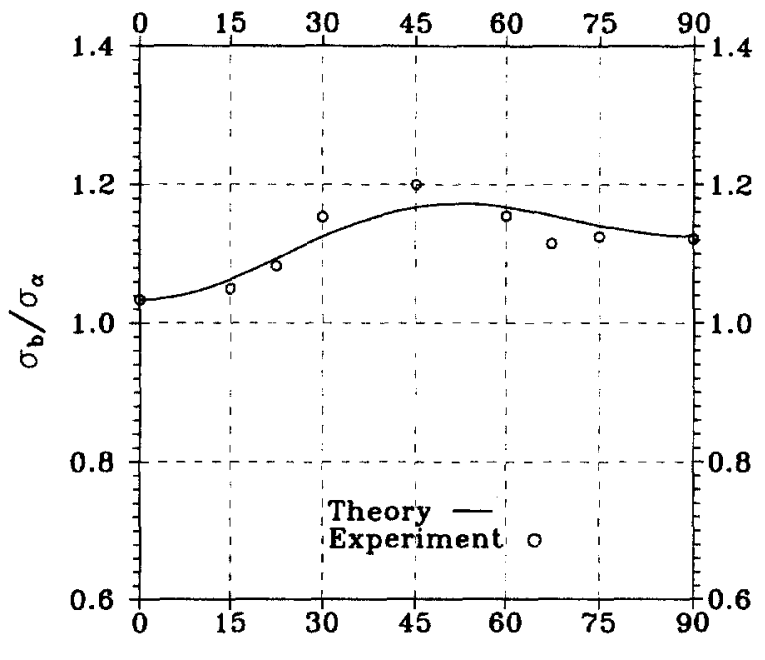

(c)

$\alpha$ (degrees)

Fig. 2. (a) Yield locus for annealed $\mathrm{A} 1100 \mathrm{P}$ aluminum sheet. (b) Plastic strain ratio, $R_{x}$, for annealed $1100-\mathrm{H} 24$ aluminum sheet. (c) Yield stress for tension specimen, $\sigma_{\alpha}$, for Annealed A1100P aluminum sheet.

and $\sigma_{45}=595 \mathrm{MPa}$. Based on these yield stresses, the following coefficients have been determined: $F=1.529 \times 10^{-6}(\mathrm{MPa})^{-2}, G=1.416 \times 10^{-6}(\mathrm{MPa})^{-2}, H=1.909 \times 10^{-6}(\mathrm{MPa})^{-2}$ and $2 N=$ $10.609 \times 10^{-6}(\mathrm{MPa})^{-2}$. The yield locus thus determined is plotted in Fig. 4(a) together with experimental data. The parameters used to determine $R_{\alpha}$ and $\sigma_{x}$ are listed in Table 3 for $\alpha=0,15$, $22.5,30,45,60,67.5,75$ and $90^{\circ}$. The results for $R_{\alpha}$ and $\sigma_{\mathrm{b}} / \sigma_{x}$ are also listed in the table and plotted in Fig. 4(b) and (c). For this material, $R>1$ and, from Fig. 4(b) and (c), it is seen that the yield stresses are almost the same for $\alpha=0^{\circ}$ and $90^{\circ}$, while the strain ratios are markedly different. 


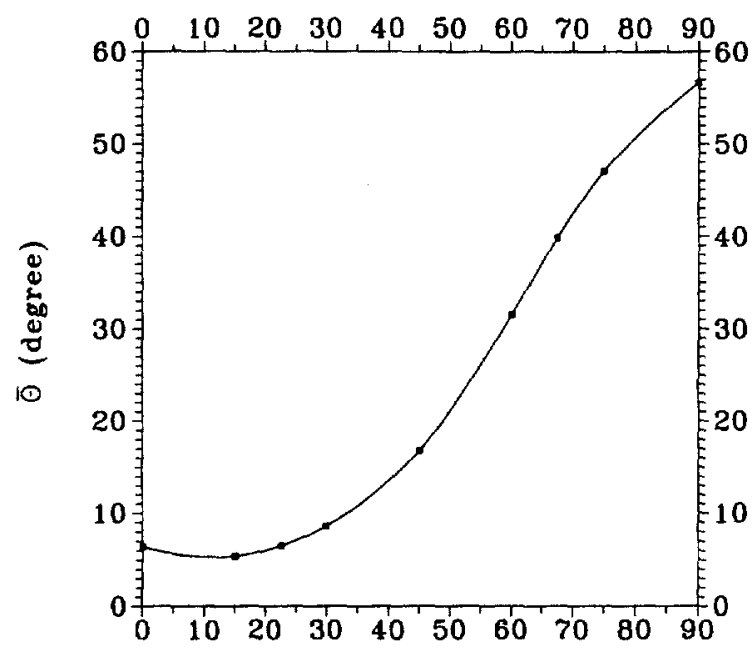

(a) $\alpha$ (degree)

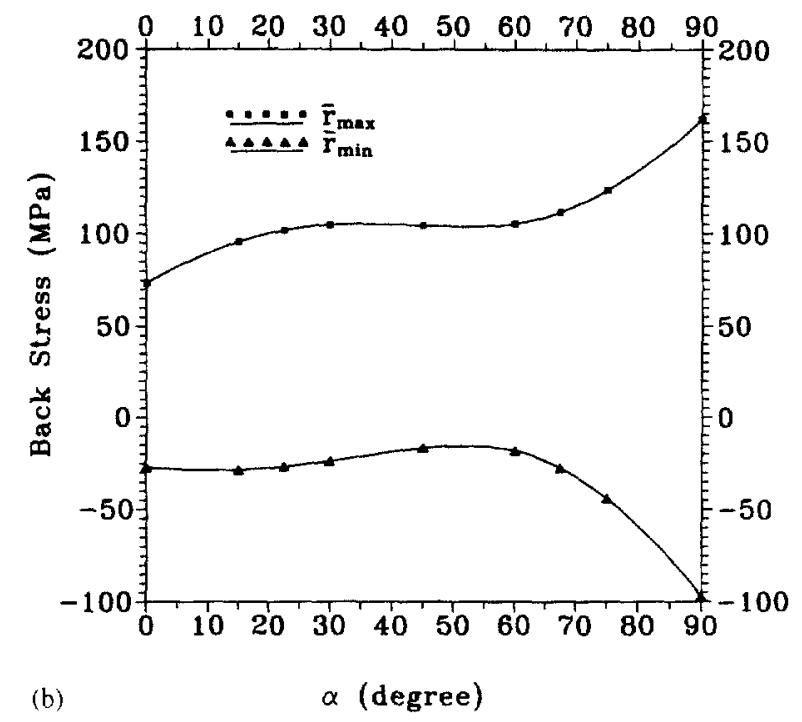

Fig. 3. (a) Principal direction $\bar{\theta}$ as a function of $\alpha$ for annealed A1100P aluminum sheet. (b) Principal back stresses $\bar{r}_{\max }$ and $\bar{r}_{\min }$ as a function of $\propto$ for annealed Al100P aluminum sheet.

Fig. 5(a) shows the principal angle $\bar{\theta}$ with respect to $\alpha$; and Fig. 5(b) shows the principal back stresses $\bar{r}_{\max }$ and $\bar{r}_{\min }$ as a function of $\alpha$. Fig. 6 shows the first and second-stage back stresses plotted in stress sub-spaces for various $\alpha$ values. Fig. 6(a) is for the annealed aluminum and Fig. 6(b) for SPCE steel.

A remark is in order. The experiments to determine the $R_{\alpha}$ ratios have not been carefully conducted. Using specimens cut at different angles $\alpha$, tension tests are performed on the specimens. It is known that due to the thinness of the specimen, the thickness strain cannot be determined with the same degree of accuracy as the longitudinal or width strain. An alternate method is related to the measurement of axial and width strains during the test. Then, the thickness strain is determined by the assumption of volume constancy. Thus,

$$
\frac{\mathrm{d} \varepsilon_{y^{\prime}}}{\mathrm{d} \varepsilon_{x^{\prime}}}=g\left(\varepsilon_{x^{\prime}}\right)
$$

where $g\left(\varepsilon_{x^{\prime}}\right)$ is measured as a function of the axial strain $\varepsilon_{x^{\prime}}$ and the width strain is denoted by $\varepsilon_{y^{\prime}}$. In this consideration, the elastic strain has been neglected, which has a significant effect when $R_{x}$ is determined at initial yielding. The volume constancy gives

$$
\mathrm{d} \varepsilon_{x^{\prime}}+\mathrm{d} \varepsilon_{y^{\prime}}+\mathrm{d} \varepsilon_{z^{\prime}}=0
$$

where $\varepsilon_{z^{\prime}}$ is the thickness strain with $z^{\prime}=z$. Using Eqs. (31) and (32), it may be shown that

$$
R=\frac{\mathrm{d} \varepsilon_{y^{\prime}}}{\mathrm{d} \varepsilon_{z^{\prime}}}=-\frac{g}{1+g}
$$



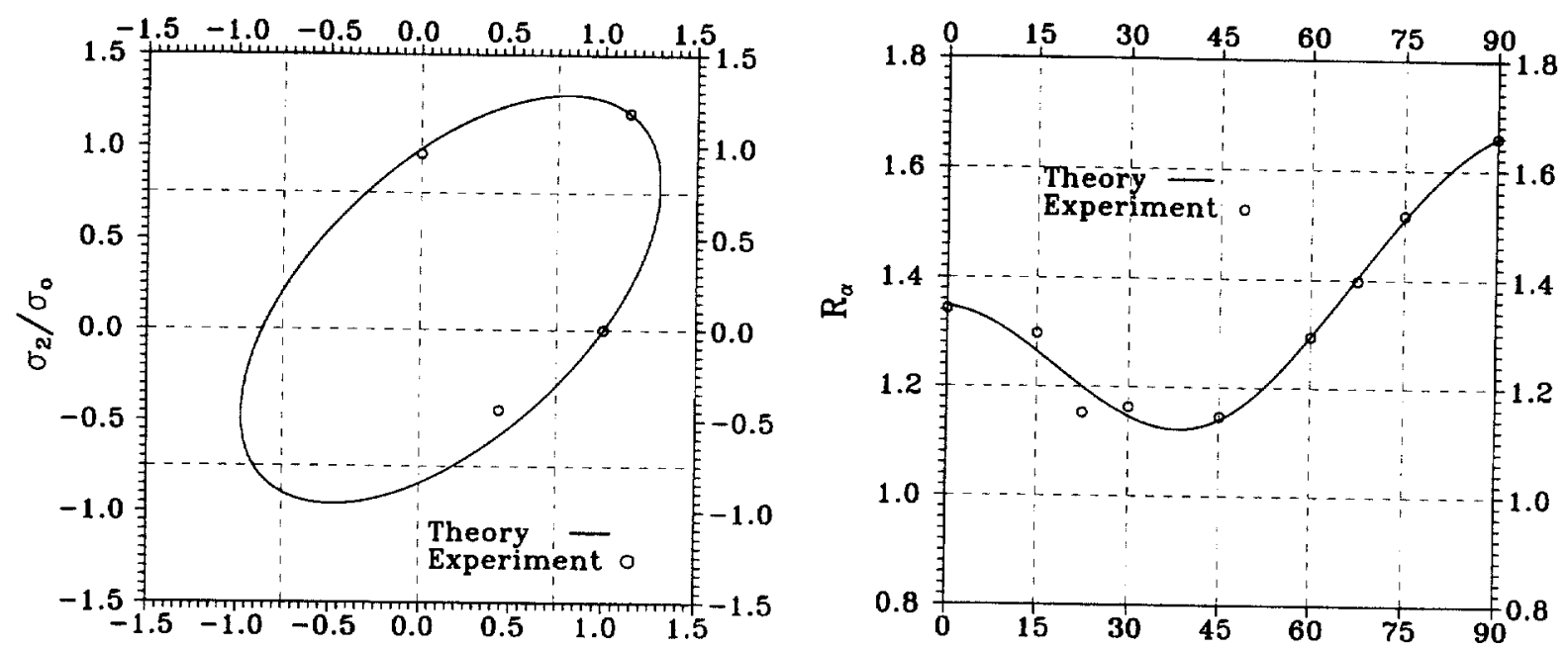

(a)

$$
\sigma_{1} / \sigma_{0}
$$

(b)

$\alpha$ (degrees)

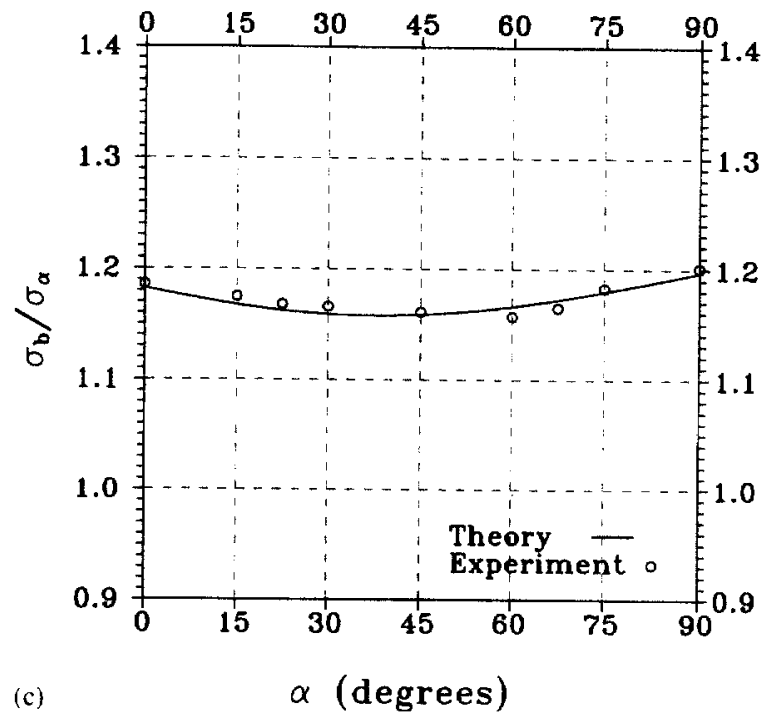

Fig. 4. (a) Yield locus for SPCE steel sheet. (b) Plastic strain ratio, $R_{\alpha}$, for SPCE steel sheet. (c) Yield stress for tension specimen, $\sigma_{x}$, for SPCE steel sheet.

The current knowledge of the $R_{x}$ ratios indicates that this ratio varies with the increasing plastic strain. But, no conclusive trend for this variation has been experimentally established. Further experiments are therefore warranted. It is difficult to determine the $R_{\alpha}$ ratio at the elastic-plastic transition zone of the stress-strain curve. However, the $R_{\alpha}$ ratios at larger plastic-strain can be systematically determined. It is important since many applications of the sheet metals are in the large plastic strain range. 


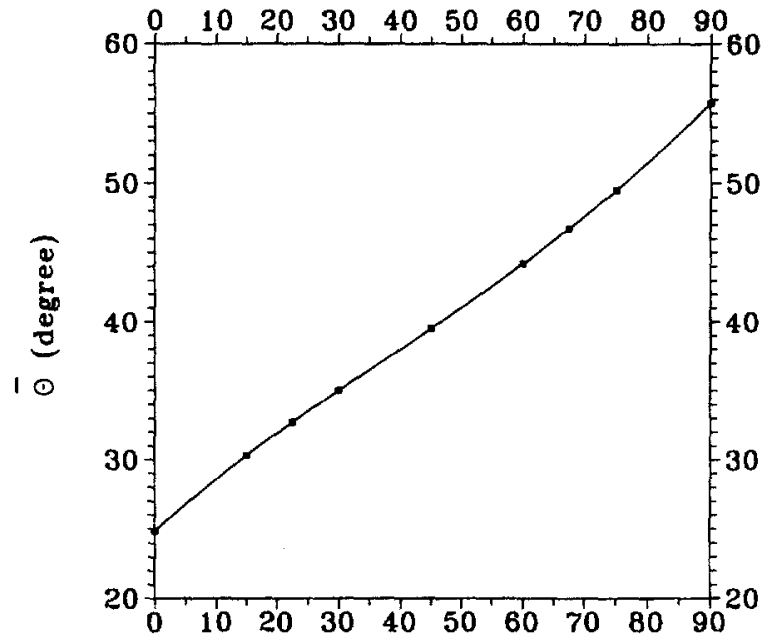

(a)

$$
\alpha \text { (degree) }
$$

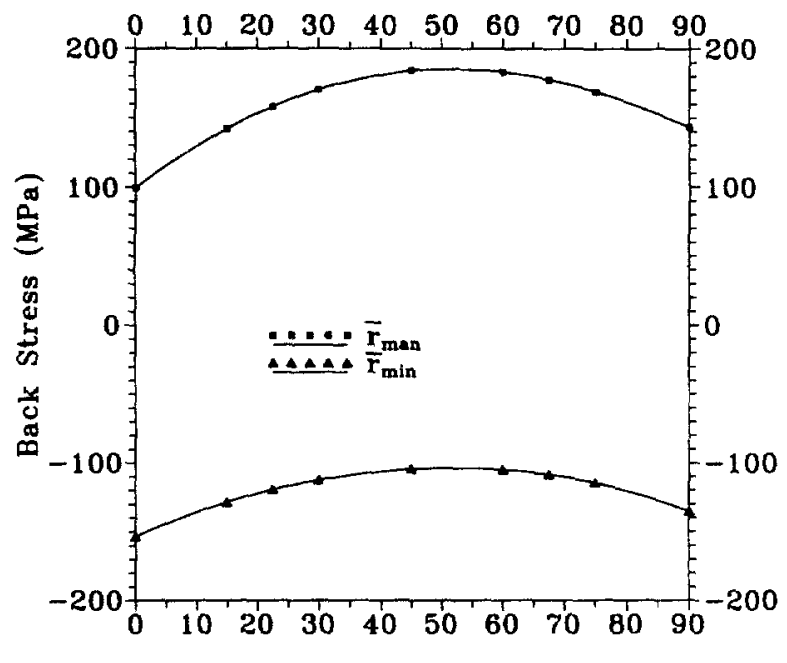

(b)

$\alpha$ (degree)

Fig. 5. (a) Principal direction $\bar{\theta}$ as a function of $\alpha$ for SPCE steel sheet. (b) Principal back stresses $\bar{r}_{\max }$ and $\vec{r}_{\min }$ as a function of $\alpha$ for SPCE steel sheet.

If the $R_{x}$ ratios are determined at a predetermined plastic strain, then the strain hardening of the tension specimens due to the tension tests should be properly accounted for in the theoretical expressions for the $R_{\alpha}$ ratios. To account for the strain hardening behavior, a hardening rule should be used.

\section{Concluding remarks}

Hill's 1948 anisotropic theory has been discussed and extended in this paper. The expanded theory has been shown to adequately describe the $R$-ratios for sheet metals, provided that information related to experiments to determine the $R$-ratio is properly incorporated into the equations. This theory has the following characteristics:

(1) A unified theory of anisotropic plasticity. The extended form of Hill's 1948 anisotropic theory is applicable to all product forms of a given metal, including sheet metal. In the case of initially isotropic material, the unified theory would reduce to the classical theory of plasticity.

(2) Quadratic yield function and normality condition for the flow rule. This theory uses Eq. (13) and its generalized form within the framework of flow theory of plasticity, where the plastic strain rate is assumed to be normal to the yield locus. The quadratic yield function given by Eq. (13) is valid for materials with all $R$ values and the more complex non-quadratic form is not needed.

(3) The coefficients of yield function are determined based on the yield stresses and not on the plastic strain ratios. Although the yield stress has several definitions, i.e. the proportional limit, proof strain, or backward extrapolation, as long as the definition is chosen, 


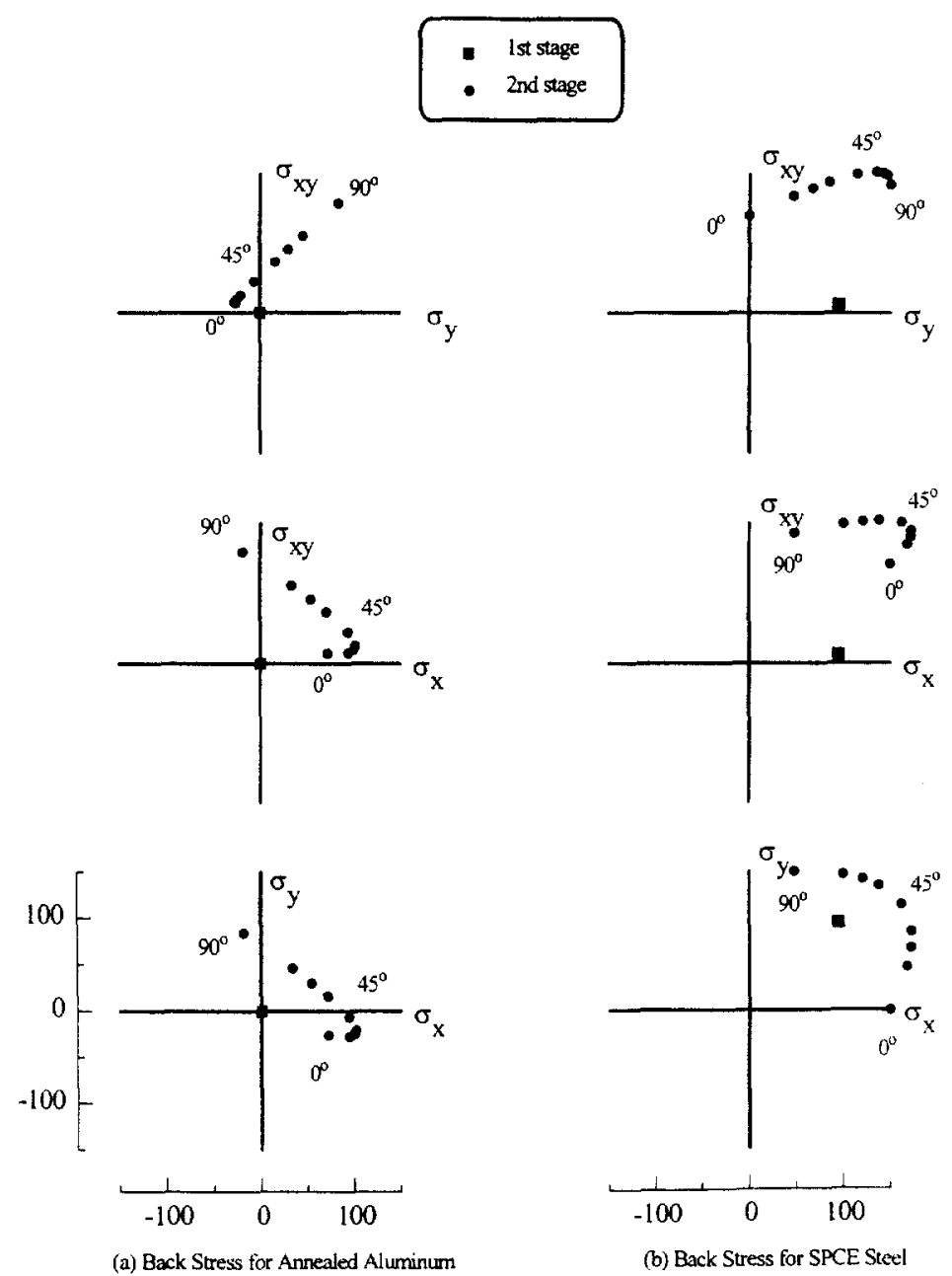

Fig. 6. (a) The first- and second-stage back stress for annealed aluminum. (b) The first- and second-stage back stress for SPCE steel.

a rather well-defined yield stress can be determined from the experiments, and the result is rather repeatable although subjected to some degree of expected experimental scatter. On the other hand, the method of determining the coefficients of yield function by use of the $R$-ratios does not conform to the traditional method of plasticity. Traditionally, the yield function is determined from the yield stresses and not from the flow rule. Since $R$ is determined by the flow rule, the coefficients of the yield function expressed in terms of $R$ would have the same effect as expressed in terms of the flow rule.

(4) The concept of isotropic and kinematic hardening is incorporated into the theory. The current theory for sheet metals does not use this concept. Using this concept, the "anomalous behavior" is just a consequence of kinematic hardening.

(5) The kinematic hardening can be used to characterize the state of rolling textures. The kinematic hardening plays an important role in the analysis of sheet metals. Since both the 
rolling textures of the sheet and the back stress evolve with the thickness reduction of the metal sheet, a correlation between the textures and the back stress can be established. This information can then be incorporated into Eqs. (13) and (14) for the prediction of subsequent plastic deformation.

\section{Acknowledgements}

This research was supported by the U.S. National Science Foundation (DMI 97-00103) and by the ROC National Science Council (NSC 85-2811-E002-022). The helpful discussions by Professor J. Chakrabarty are gratefully appreciated.

\section{References}

[1] Hill R. A theory of yielding and plastic flow of anisotropic metals. Proceedings of the Royal Society, London, 1948;A 193:281-297.

[2] Hill R. A user-friendly theory of orthotropic plasticity in sheet metals. International Journal of Mechanical Sciences 1993;35:19-25.

[3] Pearce R. Some aspects of anisotropic plasticity in sheet metals. International Journal of Mechanical Sciences 1968; 10:995-1005.

[4] Woodthorpe J, Pearce R. The anomalous behavior of aluminum sheet under balanced biaxial tension. International Journal of Mechanical Sciences 1970;12:341-347.

[5] Hill R. Theoretical plasticity of textured aggregates. Proceedings of the Cambridge Philosophical Society 1979;85:179-191.

[6] Hill R. Constitutive modelling of orthotropic plasticity in sheet metal. Journal of Mechanics and Physics of Solids 1990;38:405-417.

[7] Hosford W F. On yield loci of anisotropic cubic metals. 7th North American Metalworking Conf., SME, Dearborn MI, 1979, pp. 191-197.

[8] Gotoh M. A theory of plastic anisotropy based on a yield function of fourth order. International Journal of Mechanical Sciences 1977;19:505.

[9] Barlat F, Lian J. Plastic behavior and stretchability of sheet metals, Part 1, A yield function for orthotropic sheet under plane stress conditions. International Journal of Plasticity 1989;5:51-66.

[10] Chakrabarty J. A new yield function for planar anisotropy in sheet metal, Advances in Materials and Processing Technologies, City University, Dublin, 1993.

[11] Hill R, Hecker SS, Stout MG. An investigation of plastic flow and differential work hardening in orthotropic brass tubes under fluid pressure and axial load. International Journal of Solids Structures 1994;31:2999-3021.

[12] Taylor GI. Plastic strain in metals. Journal of Instruments and Metals 1938;62:307-324.

[13] Bishop JWF, Hill R. A theoretical derivation of the plastic properties of a polycrystalline face-centered metal. Philosophical Magazine 1951;42:414 427 and 1298-1307.

[14] Barlat F, Richmond $O$. Prediction of tricomponent plane stress yield surfaces, associated flow and failure behavior of strongly textured f.c.c. polycrystalline sheets. Materials Sciences and Engineering 1987:95:15-29.

[15] Lege DJ, Barlat F, Brem J. Characterization and modelling of the mechanical behavior and formability of a 2008-T4 sheet sample. International Journal of Mechanical Sciences 1989;31:549-563.

[16] Naruse K. Dodd B, Motoki Y. Evaluation of yield criteria for planar anisotropy in sheet metals using experimental results. In Advances in Engineering Plasticity and Its Applications, Proceedings of the Asia-Pacific Symposium on Advances in Engineering Plasticity and Its Applications - AEPA '92, Hong Kong, ed. Lee WB. 1993, pp. 235-242. 
[17] Makinde A, Thibodeau L, Neale KW. Development of an apparatus for biaxial testing using cruciform specimens. Experimental Mechanics 1992;32:138.

[18] Lin SB, Ding JL. Experimental study of the plastic yielding of rolled sheet metals with the cruciform plate specimen. International Journal of Plasticity 1995;11:583-603.

[19] Mellor PB. Experimental studies of plastic anisotropy in sheet metal. In Mechanics of Solids, The Rodney Hill 60 Anniversary Volume, ed. Hopkins HG, Sewell M. 1982, p. 383.

[20] Prager W. The theory of plasticity: a survey of recent achievements James Clayton Lecture, Proceedings of the Institute of Mechanical Engineers 1955;169:41-57. 